\title{
Worm addendum thickness and gear curvature interference for enveloping cylindrical worm drive with arc-toothed worm
}

\author{
Xinyue Zhu ${ }^{1}$, Yaping Zhao ${ }^{2}$, Yifei $\mathrm{Chi}^{2}$, Gongfa $\mathrm{Li}^{3}$, and Xinyuan $\mathrm{Chen}^{3}$ \\ ${ }^{1}$ Northeastern University College of Information Science and Engineering \\ ${ }^{2}$ Northeastern University China \\ ${ }^{3}$ Wuhan University of Science and Technology
}

April 17, 2021

\begin{abstract}
The purpose of this paper is to provide the calculation methods on worm addendum thickness and curvature interference limit line, and find the feasible value range of the technological crossing angle to avoiding addendum sharpening and curvature interference for enveloping cylindrical worm drive with arc-toothed worm. In accordance with the features of the proposed worm, the mathematical models of cutting and working are established. Based on this, the tooth profile geometry of the worm in its axial section and the worm addendum thickness are obtained by geometric analysis and calculation, and then, the feasible value range of the technological crossing angle is given. In virtue of vector rotation and elimination method, the nonlinear equation with one variable for solving the interference limit line is determined. In the process of solving nonlinear equation, the method of geometric construction is used to judge the existence of solutions and provide an initial value for the subsequent iterative calculation. The numerical example results show that with the increases of the technological crossing angle, the interference limit line is close to the boundary line of the conjugate region of the worm pair, and the hazard of curvature interference evident increases. Generally, a smaller value of the technological crossing angle within its available value range can completely avoid the occurrence of the curvature interference.
\end{abstract}

\section{Hosted file}

Worm addendum thickness and gear curvature interference.pdf available at https:// authorea.com/users/408406/articles/518432-worm-addendum-thickness-and-gear-curvature-

interference-for-enveloping-cylindrical-worm-drive-with-arc-toothed-worm 